INRA Prod. Anim., 2005, 18 (3), 153-158

\section{Le phosphore : sources, flux et rôles pour la production végétale et l'eutrophisation}

\author{
P. CASTILLON
}

Arvalis - Institut du végétal, F-31450 Baziège

Courriel : p.castillon@arvalisinstitutduvegetal.fr
Présent partout sur la terre le phosphore y est néanmoins peu concentré en général, et sa relative rareté par rapport aux besoins des êtres vivants lui confère le statut d'élément stratégique pour la vie dans le monde. En effet, sa faible biodisponibilité dans la majorité des sols cultivés en fait souvent l'élément primordial pour la satisfaction des besoins alimentaires des végétaux, et par conséquent de ceux de l'humanité. Malgré cela, les rejets intempestifs dans le réseau hydrographique conduisent dans de nombreux pays à l'eutrophisation de certains milieux aquatiques.

\section{1 / Sources de phosphore et principaux usages de cet élément}

Onzième des éléments dans l'ordre de leur abondance dans la croûte terrestre, le phosphore (P) est présent partout, mais à faible concentration dans beaucoup de roches. La masse totale de $\mathrm{P}$ dans la planète est estimée à $10^{19}$ tonnes dont $10^{15}$ tonnes dans l'écorce terrestre, $10^{11}$ tonnes dans les mers et océans et $10^{10}$ tonnes dans les sols dont les teneurs varient de 0,01 \% pour les plus dépourvus à $0,3 \%$ pour les plus fortement enrichis. Toutefois les gisements suffisamment riches pour justifier leur exploitation sont présents sur tous les continents. D’origine sédimentaire pour plus de $90 \%$ de la ressource et métamorphique pour le reste, ces gisements sont constitués de minerais, de nature apatitique pour l'essentiel, dont les teneurs en $\mathrm{P}$ varient de 10 à $17 \%$. Cette ressource qui correspond aux quantités de phosphore potentiellement exploitable s'est accrue depuis un siècle au gré des découvertes successives (figure 1), et de nouvelles découvertes ne sont pas à exclure. Elle est estimée actuellement à environ 100 milliards de tonnes de minerais qui, au
Figure 1. Evolution des ressources mondiales de minerais phosphatés (EFMA 2000).

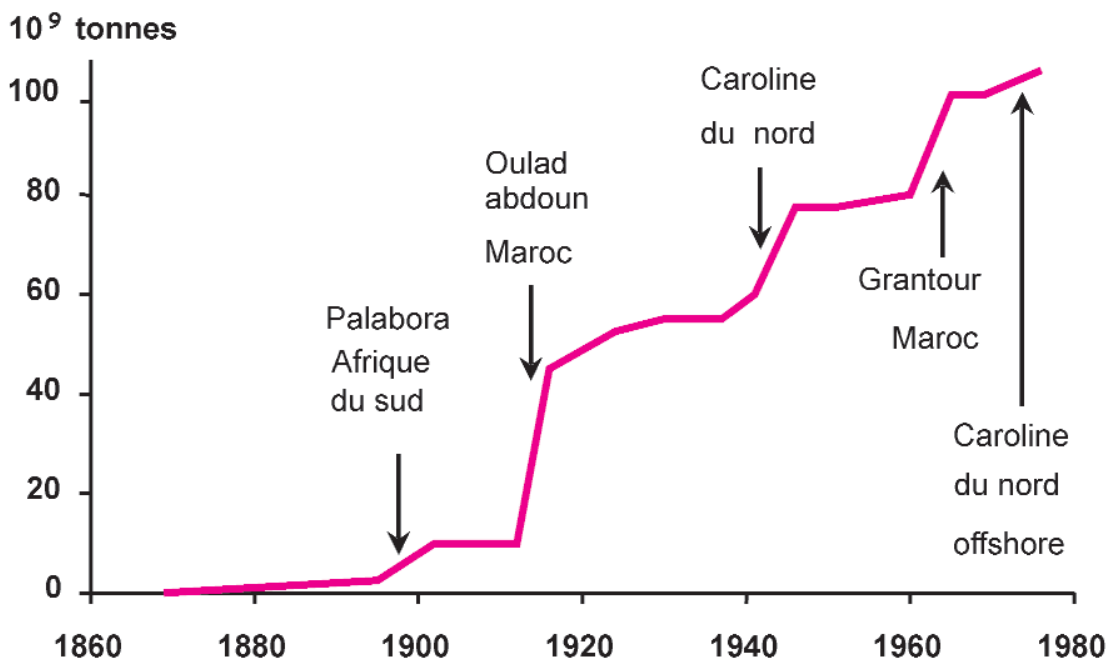

rythme actuel de consommation, sible des engrais et surtout par le recydevraient permettre de couvrir les clage du phosphore contenu dans cerbesoins mondiaux pendant 600 à tains déchets, tout particulièrement 1000 ans. Toutefois seules les réserves, les boues, les déchets urbains et les c'est-à-dire la fraction de la ressource effluents d'élevages. Le recyclage accessible à des coûts jugés accepta- deviendra certainement la seule possibles, sont actuellement exploitées. bilité de satisfaire les besoins de l'huElles concernent un petit nombre de manité lorsque les réserves ne suffiront pays (figure 2) et devraient permettre plus. de satisfaire le niveau actuel de consommation mondiale pendant environ une centaine d'années. Leur durée de vie pourrait être grandement accrue par l'utilisation la plus rationnelle pos-

L'essentiel de la production de minerai et d'acide phosphorique (base de la chimie «fine» du phosphore) est l'apanage de quelques pays et plus de la

Figure 2. Répartition des réserves de phosphates dans le monde (US Geological Survey 2001).

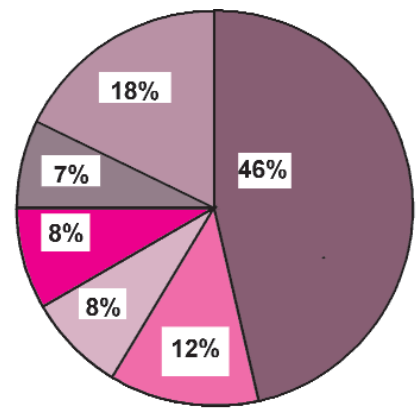

Réserves accessibles à $<36 \$ / t$ 13,5 milliards de tonnes
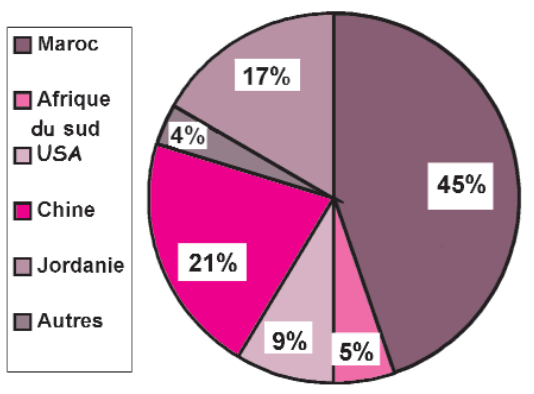

Réserves accessibles à $<90 \$ / t$ 52 milliards de tonnes 
moitié est assurée par le Maroc et les USA. Bien que les usages du phosphore soient nombreux (alimentation humaine, peintures et émaux, céramiques, pigments, électronique, pharmacie ...), 97 \% de la production mondiale sont dévolus à trois usages principaux : la production d'engrais qui à elle seule en consomme $79 \%$ en Europe et $80 \%$ à l'échelle mondiale, la production des aliments du bétail (11 \% en Europe et $5 \%$ dans le monde) et la fabrication des détergents (7\% en Europe et $12 \%$ dans le monde). Après avoir augmenté régulièrement jusqu'en 1989 pour atteindre 16,4 millions de tonnes de $\mathrm{P}$, la consommation mondiale d'engrais phosphatés a légèrement décru depuis. La consommation européenne a atteint son apogée plus tôt, en 1974 avec 1,3 millions de tonnes de P, mais la part de la consommation mondiale qu'elle représente n'a cessé de décroître depuis 1960 pour devenir inférieure à $10 \%$ actuellement. Malgré cela, les niveaux de fertilisation phosphatée demeurent encore élevés dans l'agriculture européenne et tout particulièrement en France avec un niveau moyen de $15 \mathrm{~kg} \mathrm{P} \mathrm{ha}^{-1}$ de SAU et $21 \mathrm{~kg} \mathrm{P} \mathrm{ha}^{-1}$ pour les cultures annuelles ce qui représentait 315000 tonnes de $\mathrm{P}$ en 2003-2004.

\section{2 / Rôle du phosphore pour la production végétale}

Les pratiques de fertilisation en vigueur en France depuis plusieurs dizaines d'années ont contribué à enrichir en phosphore la plupart des terres cultivées. La biodisponibilité du phosphore y est devenue telle qu'elle pourrait permettre la suppression des apports d'engrais phosphatés dans bien des cas. Malgré cela, la fertilisation systématique des cultures est bien ancrée dans la pratique de la majorité des agriculteurs qui souhaitent, par assurance, maintenir dans leurs sols la «richesse» accumulée. L'apport d'engrais phosphaté est toutefois nécessaire dans certains champs parce que la biodisponibilité du phosphore dans le sol y est insuffisante eu égard à «l'exigence» de l'espèce cultivée et à l'objectif de production qui lui est assigné ou parce que les conditions de sol et de climat sont défavorables à la croissance des racines. Il existe par ailleurs des sols cultivés qui n’ont pas bénéficié de la prodigalité des fertilisations passées. Dans ce type de situation, le phosphore des engrais joue un rôle fondamental pour la nutrition des plantes et leur production, malgré un taux d'utilisation relativement faible par celles-ci. En effet seulement $10 \%$, en moyenne, de l'élément apporté est utilisé par la culture qui reçoit l'apport (Fardeau et Colomb 2001). Les 90 \% restant sont fixés sur la phase solide du sol dont ils contribuent à entretenir la réserve de $\mathrm{P}$ biodisponible. Néanmoins la faible fraction du P de l'engrais utilisée par les plantes au cours des premières étapes de leur développement, période pendant laquelle elles sont particulièrement sensibles à la carence en phosphore (Plénet et al 2000, Rodriguez et al 1998), contribue à satisfaire leurs besoins nutritionnels. L'accroissement de la surface foliaire qui en résulte permet l'allocation aux organes souterrains d'une quantité suffisante de substances carbonées issues de la photosynthèse et de ce fait la production de racines en abondance (Mollier 1999). Celles-ci peuvent ainsi explorer un volume de sol tel qu'elles sont capables d'y puiser la quantité de phosphore nécessaire pour satisfaire les besoins de cultures à haut niveau de production, $\mathrm{y}$ compris dans les sols les plus pauvres.

Par contre, bien que les 2/3 environ du phosphore absorbé par les plantes se retrouvent dans les grains à la récolte, la teneur en phosphore de ceux-ci est, dans la pratique, moins influencée par la fertilisation phosphatée que par la

Figure 3. Variabilité de la teneur en $P$ des grains de blé et de maïs dans 2 expérimentations de longue durée réalisées en France.
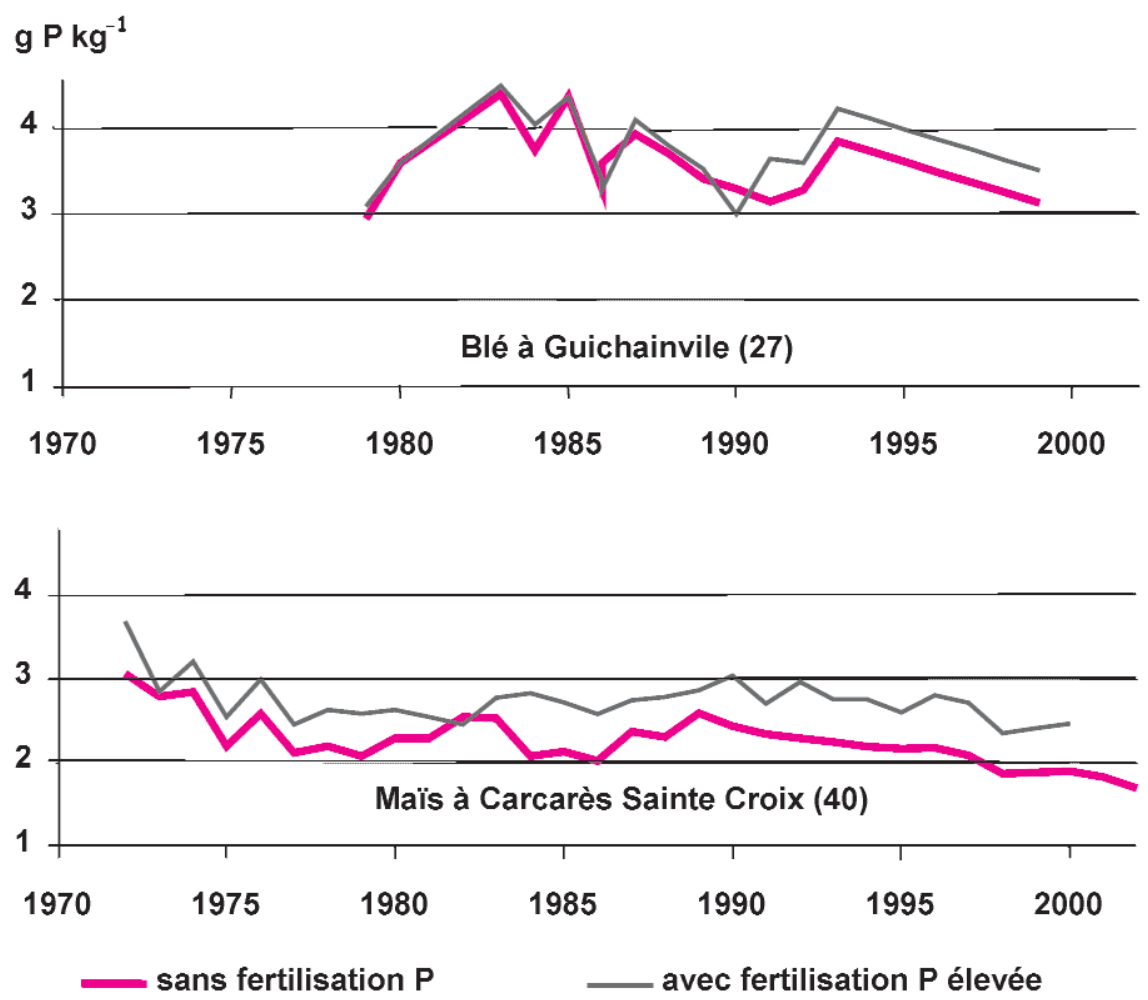

Source : Arvalis - Institut du végétal, INRA. variabilité due aux conditions climatiques et à l'espèce cultivée voire à sa variété (figure 3).

\section{3 / Rejets de phosphore dans le réseau hydrogra- phique et eutrophisation}

Les quantités de phosphore mises en jeu dans les parcelles cultivées par les apports d'engrais, minéraux ou organiques, et les exportations par les récoltes sont de l'ordre de quelques dizaines de kilogrammes par hectare, au regard desquelles les pertes par ruissellement ou drainage peuvent être considérées comme négligeables puisque celles-ci sont, en général de l'ordre de quelques centaines de grammes par ha. Néanmoins pour les sols fortement enrichis en phosphore, en raison par exemple d'apports fréquents et abondants de fumiers ou lisiers, et à l'occasion d'épisodes pluvieux particuliers, les pertes sont parfois conséquentes et atteignent $1 \mathrm{~kg}$ de $\mathrm{P}$ par ha. Elles peuvent ainsi, avec les autres sources de $\mathrm{P}$, contribuer à l'eutrophisation des milieux dans lesquels l'eau stagne ou circule lentement. Le phosphore en est l'élément déclenchant mais aussi le facteur de maîtrise (Barroin 2004). 
Figure 4. Evolution des rejets de phosphore à l'embouchure des quatre grands fleuves français.

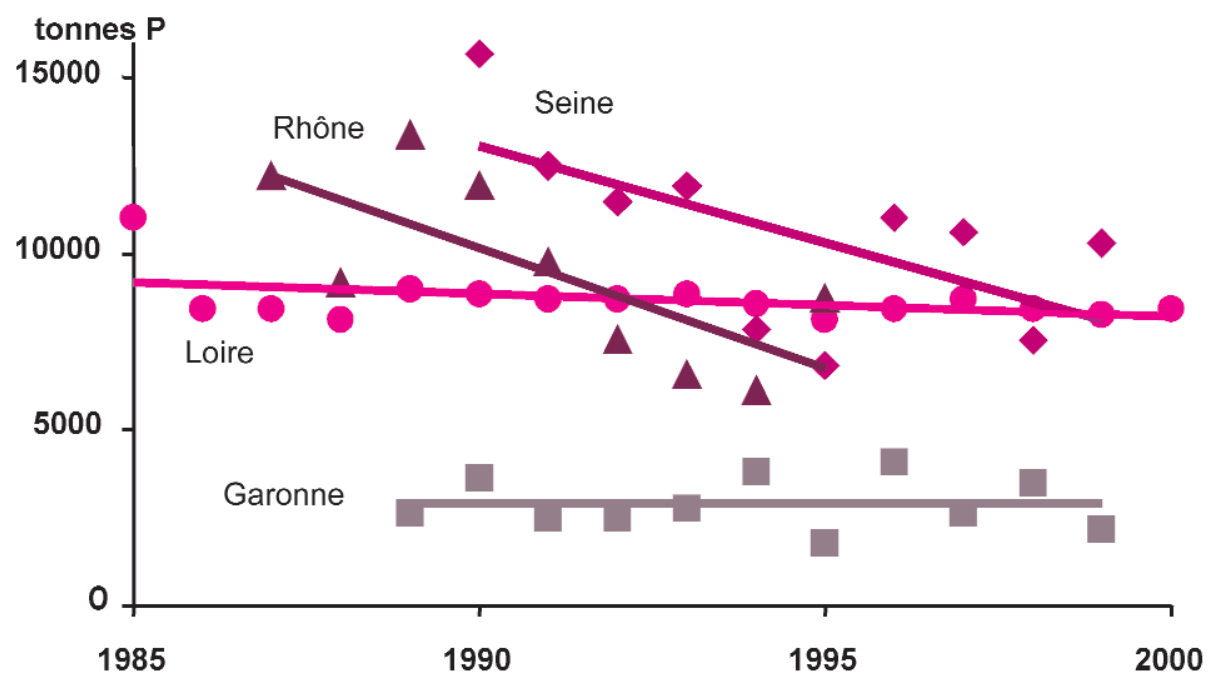

Lorsque la concentration de phosphore dans l'eau est faible, la production d'algues, socle de la chaîne alimentaire, est peu abondante et le milieu demeure oligotrophe. Lorsque la concentration de $\mathrm{P}$ dans l'eau augmente abusivement, la production végétale s'accroît fortement, avec pour corollaire une réduction de l'éclairement en profondeur, une diminution de la concentration d'azote nitrique et des épisodes d'anoxie nocturne auxquels peuvent s'ajouter de fortes augmentations diurnes du pH susceptibles d'engendrer des concentrations d'ammoniac toxiques pour le règne animal. La mortalité du poisson est la manifestation la plus spectaculaire des conséquences de l'eutrophisation. L'évolution ultime du processus conduit donc à la disparition du zooplancton et du poisson et au remplacement des algues vertes par des cyanobactéries, fixatrices de $\mathrm{N}_{2}$ et très peu consommées par le zooplancton. D'un processus de consommation caractéristique d'un milieu oligotrophe propre aux multiples usages de l'eau, l'eutrophisation conduit à un processus de décomposition d'une abondante biomasse par des bactéries, caractéristique d'un milieu dont les usages sont très limités et dont la régénération implique obligatoirement le retour à une faible concentration de phosphore dans l'eau (Barroin 2003).

\section{4 / Transferts de phosphore des sols cultivés vers le réseau hydrographique}

Les rejets de $\mathrm{P}$ dans le milieu aquatique étaient en France de l'ordre de
70000 tonnes par an au début des années 2000 dont 41600 tonnes parvenaient à la mer (source : IFEN Institut Français de l'Environnement 2002). Prés de $50 \%$ étaient imputés aux rejets d'origine domestique parmi lesquels le phosphore des détergents représentait un peu plus de la moitié. A l'industrie était attribué $1 / 4$ des rejets et aux activités agricoles le quart restant, dont le phosphore provenant des élevages représentait $80 \%$.

L'estimation de la part des rejets provenant des activités agricoles, dont l'origine est principalement diffuse, est toujours un sujet de débat. Liés aux pluies qui engendrent ruissellement, érosion et drainage, les apports de phosphore d'origine agricole sont identifiés dans les études sur les bassins versants par les variations de flux de $\mathrm{P}$ et d'eau à l'exutoire de ceux-ci. Toutefois la rétention de phosphore d'origine ponctuelle par les sédiments Fontaine du Theil à Saint Léger des Prés (35).

Source : CEMAGREF. des cours d'eau en phase de tarissement et sa libération en périodes de crues pourrait conduire à imputer à l'agricole ce qui relève d'autres origines (Dorioz et al 2004). Quoi qu'il en soit, en raison de la déphosphatation de plus en plus fréquente et efficace des rejets ponctuels qui se traduit par une diminution sensible des quantités transférées à l'embouchure des grands fleuves (figure 4) la part des rejets d'origine diffuse tend à augmenter et, probablement, celle aussi liée à l'activité agricole.

Alors que les rejets ponctuels concernent pour l'essentiel du phosphore dissous (P total présent dans l'eau filtrée à $0,45 \mu \mathrm{m}$ ) dont on peut penser qu'il est potentiellement utilisable par les végétaux aquatiques, le phosphore d'origine diffuse et agricole est transféré sous les formes dissoute et particulaire. La participation de cette dernière à l'eutrophisation dépend des états chimiques du phosphore dans les particules. Par ailleurs, la part du P dissous par rapport au $P$ particulaire varie suivant le régime des crues et les modalités de transfert du $\mathrm{P}$ des parcelles cultivées vers les cours d'eau. Les mesures réalisées pendant 5 années dans un petit bassin versant de 130 ha en Ille et Vilaine, principalement dédié aux activités de polyculture élevage, ont montré la forte variabilité des quantités de $\mathrm{P}$ dissous et de la part qu'il représente dans le $\mathrm{P}$ total véhiculé à l'exutoire du ruisseau (figure 5).

Dans le cadre d'une volonté collective de réduire les quantités de phosphore rejetées dans le réseau hydrographique français, l'agriculture ne peut ignorer sa part de responsabilité et, si nécessaire, doit mettre en œuvre des solutions adaptées.

Figure 5. Lame d'eau écoulée et phosphore rejeté à l'exutoire du bassin versant de la

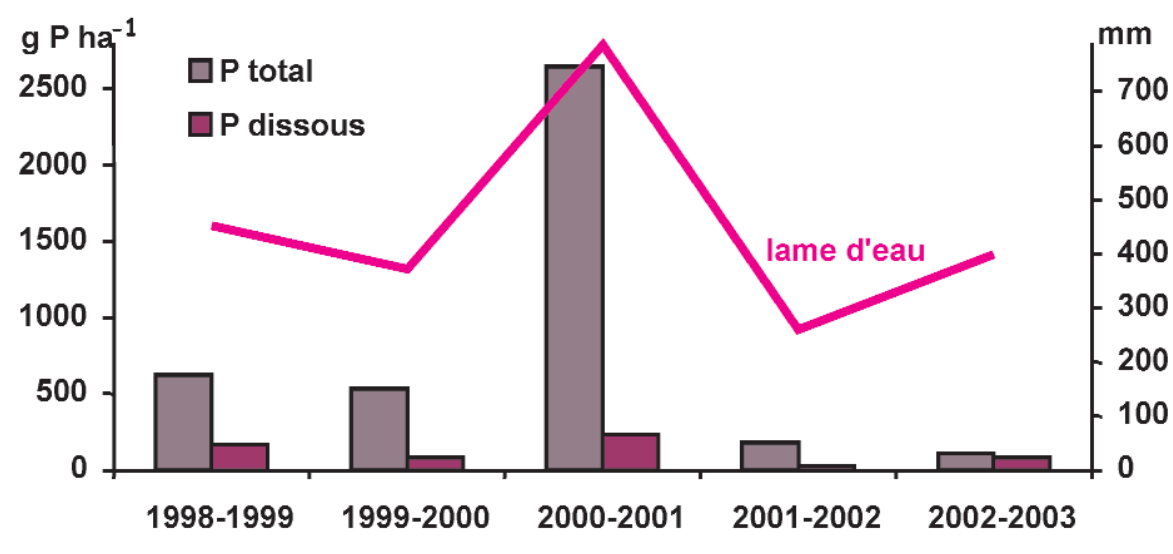




\section{5 / Limitation des transferts de phosphore d'origine agricole vers les cours d'eau}

Dans certains cas le transfert de $\mathrm{P}$ d'origine agricole vers les cours d'eau relève plus de l'apport ponctuel que du diffus. Il en est ainsi des effluents issus des bâtiments d'élevage et des aires de stockage de fumiers ou lisiers qui parviennent directement aux fossés. L'apport direct de fertilisants phosphatés dans les cours d'eau lors des épandages en bordure des parcelles peut également être assimilé à ce type d'apport.

Dans les parcelles cultivées le phosphore est transporté par les eaux de ruissellement et de drainage quel que soit le système de culture en vigueur, mais l'importance des transferts varie beaucoup selon la nature des pratiques culturales, les caractéristiques du sol, et bien entendu la lame d'eau écoulée. Celle-ci relève principalement des conditions climatiques locales et ne peut donc être maîtrisée. Par contre certaines pratiques culturales ne sont pas sans incidence sur la quantité de phosphore transféré hors des parcelles (Sharpley et al 2001).

L’occurrence d'un épisode très pluvieux après un apport d'engrais phosphaté minéral ou organique (fumier, lisier, boues ...) peut induire des pertes importantes de phosphore aussi bien par ruissellement que par drainage et engendrer des concentrations instantanées de $\mathrm{P}$ très élevées (> $1 \mathrm{mg} \mathrm{P} \mathrm{L}^{-1}$ ). Cela concerne tout par- ticulièrement les eaux des premiers épisodes de ruissellement après l'apport. Seul l'enfouissement de l'engrais dans le sol, lorsque le système de culture le permet, peut contribuer à réduire de façon significative les pertes de $\mathrm{P}$ associées à ce risque (Hart et al 2004).

Les modalités de travail du sol agissent sur le mode de circulation de l'eau et notamment sur la part respective du ruissellement et de l'infiltration en profondeur dans l'évacuation de l'excès d'eau, ainsi que sur la qualité de celleci. Par exemple, la suppression de tout travail du sol pour implanter les cultures (semis direct) contribue à réduire fortement l'érosion et par conséquent la charge des matières en suspension (MES) et de phosphore particulaire dans les eaux qui ruissellent. Cette technique induit par contre l'accumulation à la surface du sol du phosphore issu des engrais et des organes végétaux restitués. Il en découle un fort enrichissement des eaux qui ruissellent. Il s'avère par ailleurs que l'adoption de techniques de travail du sol sans labour favorise également le transfert du phosphore en profondeur et son évacuation par drainage (figure 6). Cela est probablement dû à la formation et au maintien de macropores qui favorisent le transfert rapide vers la profondeur de l'eau qui se charge de phosphore à la surface du sol.

Qu'il s’agisse des eaux de ruissellement ou de drainage, leur teneur en phosphore est étroitement dépendante de la disponibilité de cet élément dans le sol (Sharpley et Rekolainen 1997). L'enrichissement du sol en phosphore engendre donc l'augmentation de la teneur moyenne des eaux qui quittent les parcelles cultivées et réciproque- ment. Cette relation entre la teneur en phosphore du sol et celle des eaux est habituellement décrite par deux fonctions linéaires ; l'une dans le domaine des faibles teneurs en $\mathrm{P}$ du sol est caractérisée par une pente faible jusqu'à la teneur critique dénommée «Change point» au dessus de laquelle la teneur en $\mathrm{P}$ des eaux s'accroît beaucoup plus rapidement lorsque la teneur en $\mathrm{P}$ du sol augmente (Heckrath et al 1995). Cette teneur critique, qui serait supérieure à celle au-dessus de laquelle l'apport d'engrais phosphaté n'est plus nécessaire pour garantir à la plupart des espèces cultivées une alimentation non limitante pour leur production (Hart et al 2004), est souvent considérée comme seuil de référence pour la gestion des transferts de phosphore hors des parcelles cultivées. Elle semble toutefois très variable d'un type de sol à l'autre (Hesketh et Brookes 2000).

L’enjeu de la fertilisation raisonnée pourrait donc résider dans la nécessité de maintenir une disponibilité suffisante du phosphore dans les sols pour assurer aux plantes une alimentation phosphatée garante d'une production élevée mais en limitant les risques de transfert vers le réseau hydrographique. Facile à concevoir pour les systèmes de cultures «céréaliers» grâce à l'adaptation de la fertilisation strictement minérale aux caractéristiques des sols, cette démarche se heurte dans beaucoup d'exploitations d'élevage au problème de l'excédent structurel de phosphore et à l'obligation d'épandre les fumiers, lisiers et autres effluents sur les terres cultivées pour en assurer le recyclage.

Figure 6. Effet des modalités de travail du sol sur les pertes cumulées de phosphore par drainage de 1998 à 2004 ( $\mathrm{g} P$ ha-1) et sur les teneurs moyennes pondérées des eaux drainées ( $\mu m g P L^{-1}$ ) dans deux dispositifs expérimentaux sur sols limoneux.
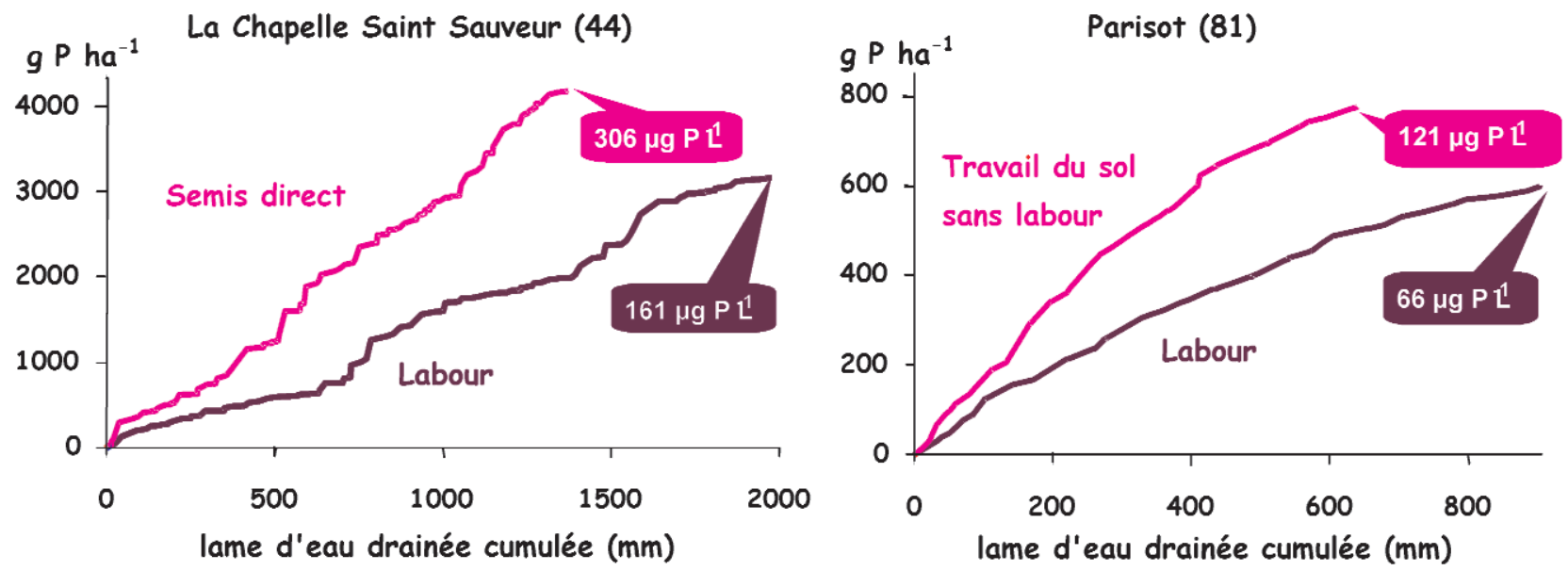


\section{6 / Gestion du phosphore des effluents dans les exploitations d'élevage}

Les lisiers, les fumiers et leurs composts sont les principaux effluents phosphorés dans les exploitations d'élevage. Le phosphore y est majoritairement présent sous des formes minérales diverses et en partie sous forme organique ou associé à celle-ci. Pour des raisons liées à la nécessité d'épandre la totalité de la ressource produite, à la facilité de gestion de celle-ci en privilégiant les apports sur les parcelles les plus proches des bâtiments d'élevage, et parce que l'attention des éleveurs a été focalisée sur l'azote à travers les contraintes environnementales associées au nitrate, les apports de phosphore par les effluents d'élevages dépassent très souvent les exportations par les cultures conduisant ainsi à l'enrichissement continu des sols dans les parcelles réceptrices.

Outre la répartition de la ressource sur la surface «épandable» la plus grande possible en tenant compte de la disponibilité du phosphore dans les sols, plusieurs alternatives sont proposées afin de limiter les risques de transfert par ruissellement et drainage. La première consiste à enfouir le plus vite possible le produit épandu dans les sols en pente pour éviter le transport du phosphore par les eaux de ruissellement dont les premières qui interviennent après l'épandage s'enrichissent le plus en phosphore (Hart et al 2004). Traiter les effluents par des sels tels la chaux ou le sulfate d'aluminium hydraté $\left(\mathrm{Al}_{2}\left(\mathrm{SO}_{4}\right)_{3}, 14 \mathrm{H}_{2} \mathrm{O}\right)$ afin de précipiter le phosphore soluble a été proposé (Smith et al 2004). Cette voie qui s'apparente au gaspillage d'une ressource limitée à l'échelle mondiale semble peu utilisée pour l'instant dans la pratique. La solution la plus judicieuse est celle qui consisterait à réduire de façon significative les rejets de phosphore par les animaux en ajustant au mieux les apports de $\mathrm{P}$ à leurs besoins et en améliorant l'efficacité de ceux-ci (Toor et al 2005). Limiter la quantité de $P$ dans le régime alimentaire ou accroître l'efficacité de celui de l'aliment de base, par l'addition de phytase par exemple, contribue à diminuer sensiblement les quantités rejetées par les animaux sans toutefois modifier la nature des formes de phosphore et par conséquent la valeur fertilisante de l'effluent (Maguire et al 2004).

\section{Conclusion}

Le phosphore des minerais représente une ressource, vitale pour l'humanité mais limitée. Les estimations actuelles évaluent à une centaine d'années la durée de vie des réserves facilement exploitables, ce qui devrait inciter à l'utilisation parcimonieuse de cet élément dont les rejets abusifs dans le réseau hydrographique engendrent par ailleurs l'eutrophisation de certains milieux aquatiques. Elément déclanchant du phénomène, le phosphore en est aussi le seul facteur de maîtrise réellement efficace à long terme.

Bien que les activités agricoles ne contribuent que de façon minoritaire pour l'instant aux rejets de $\mathrm{P}$ dans le réseau hydrographique, il est souhaitable qu'elles participent aussi à en limiter les transferts vers les cours d'eau. Ce problème concerne tout particulièrement les exploitations d'élevage en excédent structurel de phosphore qui devraient s'attacher à limiter la richesse des sols en cet élément dans les limites qui garantissent une alimentation phosphatée suffisante des cultures mais sans trop enrichir les eaux qui ruissellent ou s'infiltrent.

\section{Références}

Barroin G., 2003. Phosphore, azote et prolifération des végétaux aquatiques. Courrier Env. INRA, 48, 13-26.

Barroin G., 2004. Phosphore, azote, carbone.... Du facteur limitant au facteur de maîtrise. Courrier Env. INRA, 52, 23-30.

Dorioz J.M., Quetin P., Lazzarotto J., Orand A., 2004. Bilan du phosphore dans un bassin versant du lac Léman : conséquences pour la détermination de l’origine des flux exportés. Rev. Sci. Eau, 17 (3), 329-354.

EFMA, 2000. Phosphorus essential element for food production. European Fertilizer Manufacturers’ Association, Brussels, Belgium.

Fardeau J.C., Colomb B., 2001. Fertilisation phosphatée et potassique raisonnées. Quels défis face aux besoins des filières et aux enjeux territoriaux ? In : Actes des $5^{\mathrm{e}}$ Rencontres de la fertilisation raisonnée et de l'analyse de terre. Blois, France, 83-97.

Hart M.R., Quin B.F., Long Nguyen M., 2004. Phosphorus runoff from agricultural land and direct fertilizer effects: a review. J. Env. Quality, 33, 1954-1972.

Heckrath G., Brookes P.C., Poulton P.R., Goulding K.W.T., 1995. Phosphorus leaching from soils containing different phosphorus con- centration in the Boadbalk experiment. J. Env. Quality, 24, 904-910.

Hesketh N., Brookes P.C., 2000. Development of an indicator for risk of phosphorus leaching. J. Env. Quality, 29, 105-110.

Maguire R.O., Sims J.T., Saylor W.W., Turner B.L., Angel R., Applegate T.J., 2004. Influence of phytase addition to poultry diets on phosphorus forms and solubility in litters and amended soils. J. Env. Quality, 33, 23062316.

Mollier A., 1999. Croissance racinaire du maïs (Zea mays L.) sous déficience en phosphore. Etude expérimentale et modélisation. Thèse Université Paris Sud, 194p.

Plénet D., Etchebest S., Mollier A., Pellerin S., 2000. Growth analysis of maize field crops under phosphorus deficiency. I. Leaf growth. Plant and Soil, 223, 117-130.

Plénet D., Mollier A., Pellerin S., 2000. Growth analysis of maize field crops under phosphorus deficiency. II. Radiation use efficiency, biomass accumulation and yield components. Plant and Soil, 224, 259-272.

Rodriguez D., Keltjens W.G., Goudrian J., 1998. Plant leaf area expansion and assimilate production in wheat (triticum aestivum L.) growing in low phosphorus conditions. Plant and Soil, 200, 227-240.

Sharpley A.N., Rekolainen S., 1997. Phosphorus in agriculture and its environmental implications. In: Phosphorus loss from soil to water. CAB International (Eds), 1-53.

Sharpley A.N., McDowell R.W., Kleinman P.J.A., 2001. Phosphorus loss from land to water: integrating agricultural and environmental management. Plant and Soil, 237, 287-307.

Smith D.R., Moore P.A., Miles D.M., Haggard B.E., Daniel T.C., 2004. Decreasing phosphorus runoff losses from land applied poultry litter with dietary modifications and Alum addition. J. Env. Quality, 33, 2210-2216.

Toor G.S., Sims J.T., Dou Z., 2005. Reducing phosphorus in dairy diets improves farm nutrient balances and decreases the risk of nonpoint pollution of surfaces and ground waters. Agric. Ecosystems Env. 105, 401-411.

US Geological Survey, 2001. In: www.ppifar.org. Inorganic phosphorus and potassium production and reserves. Roberts T.L., Stewart W.M., Better Crops 2002, 86 (2), 6-7. 


\title{
Résumé
}

Le phosphore constitue un élément stratégique pour la vie sur terre en raison de sa faible disponibilité dans la plupart des milieux. Les réserves mondiales, constituées de gisements de phosphates apatitiques pour l'essentiel, sont limitées. Au rythme actuel de leur exploitation elles risquent d'être épuisées dans une centaine d'années ce qui n'empêche pas des rejets intempestifs dans les cours d'eau. Ceux-ci sont la cause première de l'eutrophisation de certains milieux aquatiques dont la maîtrise passe obligatoirement par la réduction des rejets, et tout particulièrement de ceux qualifiés de ponctuels.

Malgré son caractère essentiellement diffus, le transfert de phosphore vers le réseau hydrographique imputable aux activités agricoles est loin d'être négligeable. Cela est particulièrement vrai dans les zones d'élevage dont les exploitations sont très souvent en excédent structurel de phosphore recyclé en permanence par le biais des fumiers, lisiers et autres effluents épandus sur les terres cultivées. La diminution des rejets de $\mathbf{P}$ par les animaux constitue sans doute de ce fait la principale voie par laquelle l'agriculture peut contribuer à l'effort collectif de maîtrise du problème de l'eutrophisation.

\begin{abstract}
Phosphorus : sources, flow and roles for plant production and eutrophication

Phosphorus is a strategic element for life on earth because of its low availability in most environments. World reserves, mainly consisting of apatite deposits, are limited. At the present rate of exploitation they risk being exhausted in about a hundred years, but this does not prevent surplus amounts reaching watercourses. These are the primary cause of eutrophication of certain aquatic environments, the control of which necessarily involves the reduction of emissions, and in particular those known as localised emissions.

In spite of its essentially diffuse character, phosphorus transfer into the hydrological network attributed to agricultural activities is far from negligible. This is particularly true in livestock rearing regions where the farms are very often producing a permanent excess of recycled phosphorus due to manures, slurries and other effluents which are spread on the cultivated land. Because of this, the reduction of $\mathbf{P}$ emissions from animals is undoubtedly the main way in which agriculture can contribute to the collective effort to control the problem of eutrophication.
\end{abstract}

CASTILLON P., 2005. Le phosphore : sources, flux et rôles pour la production végétale et l'eutrophisation. INRA Prod. Anim., 18, 153-158. 